\title{
Elastic and quasi-elastic electron scattering off nuclei with neutron excess
}

\author{
Andrea Meucci, Matteo Vorabbi, Carlotta Giusti, and Franco Davide Pacati \\ Dipartimento di Fisica, Università degli Studi di Pavia and \\ INFN, Sezione di Pavia, via A. Bassi 6, I-27100 Pavia, Italy \\ Paolo Finelli \\ Dipartimento di Fisica e Astronomia, \\ Università degli Studi di Bologna and \\ INFN, Sezione di Bologna, via Irnerio 46, I-40126 Bologna, Italy
}

(Dated: November 2, 2018)

We present theoretical predictions for electron scattering on oxygen and calcium isotopic chains. The calculations are done within the framework of the distorted-wave Born approximation and the proton and neutron density distributions are evaluated adopting a relativistic Dirac-Hartree model. We present results for the elastic and quasi-elastic cross sections and for the parity-violating asymmetry. As a first step, the results of the models are tested in comparison with some of the data available for elastic and quasi-elastic scattering on ${ }^{16} \mathrm{O}$ and ${ }^{40} \mathrm{Ca}$ nuclei. Then, the evolution of some nuclear properties is investigated as a function of the neutron number. We also present a comparison with the parity-violating asymmetry parameter obtained by the PREX Collaboration on ${ }^{208} \mathrm{~Pb}$ and give a prediction for the future experiment CREX on ${ }^{48} \mathrm{Ca}$.

PACS numbers: 21.60.Jz; 21.10.-k; 25.30.Fj; 24.10.Jv

Keywords: Parity-violating electron scattering; Relativistic mean field models, Ground-state properties 


\section{INTRODUCTION}

The nuclear response to an external probe is a powerful tool to investigate the structure of hadron systems such as atomic nuclei and their constituents. In particular, electronscattering reactions have provided the most complete and detailed information on nuclear and nucleon structure [1-6]. Electrons interact with nuclei predominantly through the electromagnetic interaction, which is well known from quantum electrodynamic and is weak compared with the strength of the interaction between hadrons. The scattering process is therefore adequately treated assuming the validity of the Born approximation, i.e., the one-photon exchange mechanism between electron and target. The virtual photon, like the real one, has a mean free path much larger than the target dimensions, thus exploring the whole target volume. This contrasts with hadron probes, which are generally absorbed at the target surface. Moreover, the ability to vary independently the energy $\omega$ and momentum $\boldsymbol{q}$ of the exchanged virtual photon transferred to the nucleus makes it possible to map the nuclear response as a function of its excitation energy with a spatial resolution that can be adjusted to the scale of processes that need to be studied.

Several decades of experimental and theoretical work on electron scattering have provided a wealth of information on the properties of stable nuclei. Nuclear charge density distributions and charge radii have been determined from the analysis of elastic electron scattering data $[7,8]$. At low energy loss bound nucleons may produce excited states as a result of single-particle (s.p.) transitions and/or collective motion. Spectroscopic analysis of such excitation mechanisms is the traditional field of nuclear physics. The systematic investigation of inelastic electron scattering has given the necessary support to the foundation of many-body theories applied to the nuclear systems. Spin, parities, and the strength and structure of the transition densities connecting the ground and excited states have been studied $[2,9]$. In comparison with hadron scattering, which also gives important information, only electron scattering can be used to determine the detailed spatial distributions of the charge transition densities for a variety of single-particle and collective transitions. At energy loss little above particle emission threshold the quasi-bound giant resonances occur. Here the possibility of independently varying $\omega$ and $\boldsymbol{q}$, in conjunction with the coincident detection of nucleons emitted in the decay process, allows a detailed analysis of the various types of collective motions that are responsible for contributions of different multipolarities 
to such resonances. Several electric and magnetic giant multipole resonances have been discovered and studied using electron scattering $[10,11]$.

At energies above giant resonances a large broad peak occurs at about $\omega=q^{2} /\left(2 m_{N}\right)$, where $m_{N}$ is the nucleon mass. Its position corresponds to the elastic peak in electron scattering by a free nucleon. It is quite natural to assume that a quasi-free process is responsible for such a peak with a nucleon emitted quasi-elastically. If the nucleons were indeed free, the peak would be sharp and would just occur at $\omega=q^{2} /\left(2 m_{N}\right)$, corresponding to the energy taken by the recoiling free nucleon. A shift in the position of the peak is produced by the nuclear binding, while a broadening of the peak is produced by Fermi motion.

Coincidence $\left(e, e^{\prime} p\right)$ experiments in the quasi-elastic $(\mathrm{QE})$ region represent a very clean tool to explore the proton-hole states. A large amount of data for the exclusive $\left(e, e^{\prime} p\right)$ reaction have confirmed the assumption of a direct knockout mechanism and has provided accurate information on the s.p. structure of stable closed-shell nuclei $[4,5,12-17]$. The separation energy and the momentum distribution of the removed proton, which allows to determine the associated quantum numbers, have been obtained. From the comparison between experimental and theoretical cross sections it has been possible to extract the spectroscopic factors, which give a measurement of the occupation of the different shells and, as a consequence, of the effects of nuclear correlations, which go beyond a mean field description of nuclear structure.

In the inclusive $\left(e, e^{\prime}\right)$ process only the scattered electron is detected and the final nuclear state is undetermined, but the main contribution in the region of the QE peak still comes from the interaction on single nucleons. In comparison with the exclusive $\left(e, e^{\prime} p\right)$ process, the inclusive $\left(e, e^{\prime}\right)$ scattering corresponds to an integral over all available nuclear states and consequently provides less specific information, but it is more directly related to the dynamics of the initial nuclear ground state. The width of the QE peak can give a direct measurement of the average momentum of nucleons in nuclei, the shape depends on the distribution in energy and momentum of the initially bound nucleons. Precise measurements can give direct access to integrated properties of the nuclear spectral function which describes this distribution. A considerably body of QE data for light-to-heavy nuclei in different kinematic situations has been collected $[5,6,18]$. Not only differential cross sections, but also the contribution of the separate longitudinal and transverse response functions have 
been considered. From the theoretical point of view, many efforts have been devoted to the description of the available data and important progress has been achieved in terms of experimental results and theoretical understanding [4-6].

The use of the electron probe can be extended to exotic nuclei. The detailed study of the properties of nuclei far from the stability line and the evolution of nuclear properties with respect to the asymmetry between the number of neutrons and protons is one of the major topics of interest in modern nuclear physics.

In the next years the advent of radioactive ion beams (RIB) facilities [19-21] will provide a large amount of data on unstable nuclei. A new generation of electron-RIB colliders using storage rings is under construction at RIKEN (Japan) [22-24] and GSI (Germany) [25]. These facilities will offer unprecedented opportunities to study the structure of exotic unstable nuclei through electron scattering in the ELISe experiment at FAIR in Germany [26-28] and the SCRIT project in Japan [29, 30]. Kinematically complete experiments, where, in contrast to conventional electron scattering, all target-like reaction products are detected, will become feasible for the first time, allowing a clean separation of different reaction channels as well as a reduction of the unavoidable radiative background seen in conventional experiments. Therefore, even applications using stable isotope beams will be of interest.

Several papers devoted to theoretical treatments of electron scattering off exotic nuclei have recently been published, underlining the usefulness of electron scattering for investigating the structure of unstable nuclei [31-48]. In this work we give another contribution to this field. Here we present and discuss numerical predictions for elastic and inclusive QE electron scattering cross sections on oxygen $\left({ }^{14-28} \mathrm{O}\right)$ and calcium $\left({ }^{36-56} \mathrm{Ca}\right)$ isotopic chains.

The study of the evolution of nuclear properties along isotopic chains requires a good knowledge of nuclear matter distributions for protons and neutrons separately. The ground state densities reflect the basic properties of effective nuclear forces and provide fundamental nuclear structure information. Elastic electron scattering allows to measure with excellent precision only charge densities and therefore proton distributions. It is much more difficult to measure neutron distributions. Our present knowledge of neutron densities comes primarily from hadron scattering experiments, the analysis of which requires always model-dependent assumptions about strong nuclear forces at low-energies. A model-independent probe of neutron densities is provided by parity-violating elastic electron scattering, where direct 
information on the neutron density can be obtained from the measurement of the parityviolating asymmetry $A_{p v}$ parameter, which is defined as the difference between the cross sections for the scattering of right- and left-handed longitudinally polarized electrons [4951]. This quantity is related to the radius of the neutron distribution $R_{n}$, because $Z^{0}$-boson exchange, which mediates the weak neutral interaction, couples mainly to neutrons and provides a robust model-independent measurement of $R_{n}$.

In 2012, the first measurement of $A_{p v}$ [52] (and weak charge form factor [53]) in the elastic scattering of polarized electrons from ${ }^{208} \mathrm{~Pb}$ has been performed in Hall $\mathrm{A}$ at the Jefferson Lab (experiment PREX). The PREX collaboration obtained $A_{p v}=0.656 \pm 0.060$ (stat) \pm 0.014(syst) ppm, corresponding to a difference between the radii of the neutron and proton distributions $R_{n}-R_{p}=0.33_{-0.18}^{+0.16} \mathrm{fm}$. Unfortunately with such large uncertainties it is not possible to draw definite conclusions about the radius and the distribution of neutrons in a heavy finite nucleus like ${ }^{208} \mathrm{~Pb}$. The problems that affected the original setup will be strongly reduced by improving electronics and radiation protection (see the recently approved PREXII experiment [54]). In addition, the CREX experiment [55], with the goal of measuring the neutron skin of ${ }^{48} \mathrm{Ca}$, has also been conditionally approved. The combined analysis of the PREX and CREX experiments will allow to test the assumptions of microscopic models by testing the dependence of $R_{n}$ on the atomic mass number $A$.

From a theoretical point of view, parity violation in elastic electron scattering has been recently studied by several authors within the framework of mean field approaches [43$46,56-59]$ after almost a decade from the first detailed calculations $[51,60,61]$. In particular, in Ref. [58] the authors suggested that a $1 \%$ measurement of $A_{p v}$ can constrain the slope $L$ of the symmetry energy close to a $10 \mathrm{MeV}$ level of accuracy. A precise measurement of $R_{n}$ is definitely a crucial step to improve our knowledge of neutron-rich matter, i.e., the outer part of neutron stars.

In this paper we present calculations of $A_{p v}$ performed for oxygen and calcium isotopic chains and test the isotopic dependence. In addition, we present a comparison with the results of the PREX collaboration and provide some estimates for the future experiment CREX.

The basic ingredients of the calculations for both elastic and QE scattering are the ground state wave functions of proton and neutron s.p. states, for which we have used a relativistic mean field (RMF) model. In the last years mean field approaches have been very successfully 
employed in several aspects of nuclear structure phenomena. Effective hadron field theories with medium dependent parameterizations of the meson-nucleon vertices retain the basic structure of the relativistic mean-field framework, but can be more directly related to the underlying microscopic description of nuclear interactions [62-64]. After the first applications $[65,66]$, restricted to infinite systems and spherical nuclei, with the parameterizations DD-ME1 [67] and DD-ME2 [68], calculations have been extended to open shell nuclei, exotic systems, superheavies, and collective resonances [69].

The cross sections for elastic electron scattering are obtained from the numerical solution of the partial wave Dirac equation and includes Coulomb distortion effects.

In inclusive QE electron scattering a proper description of the final-state interactions (FSI) between the emitted nucleon and the residual nucleus is an essential ingredient for the comparison with data. For the calculations presented in this paper we have employed the relativistic Green's function model, which has been widely and successfully applied to the analysis of QE electron and neutrino-nucleus scattering data on different nuclei [70-77].

As a first step, we test the results of the models in comparison with some of the data available for elastic and QE scattering on ${ }^{16} \mathrm{O}$ and ${ }^{40} \mathrm{Ca}$ nuclei. Then, with extensive calculations on oxygen and calcium isotopic chains, we investigate the evolution of some ground-state properties as a function of the neutron number.

The case of the exclusive quasi-free $\left(e, e^{\prime} p\right)$ reaction has been investigated in $[47,78]$, where the cross sections obtained with different relativistic and nonrelativistic approaches based on the mean-field description for the proton bound state wave function are compared for oxygen and calcium isotopic chains.

The paper is organized as follows. In Sec. II we give the basic formalism involved in the description of elastic, quasi-elastic, and parity-violating electron scattering. In Sec. III we present a brief discussion of the relativistic mean-field model and of the calculations of the self-consistent ground-state proton and neutron densities of calcium and oxygen isotopes. In Sec. IV we outline the main features of the relativistic Green's function model, which is used to describe final-state interactions in the inclusive quasi-elastic electron scattering. In Sec. $\mathrm{V}$ we show and discuss our theoretical results obtained for elastic, quasi-elastic, and parity-violating electron scattering. Finally, in Sec. VI we summarize our results and present our conclusions. 


\section{ELASTIC AND QUASI-ELASTIC ELECTRON SCATTERING}

\section{A. Elastic electron scattering}

In the one-photon exchange approximation and neglecting the effect of the nuclear Coulomb field on incoming and outgoing electrons, i.e. in the plane-wave Born approximation (PWBA), the differential cross section for the elastic scattering of an lectron with momentum transfer $q$ off a spherical spin-zero nucleus is given by

$$
\left(\frac{\mathrm{d} \sigma}{\mathrm{d} \Omega^{\prime}}\right)_{E L}=\sigma_{M}\left|F_{p}(q)\right|^{2},
$$

where $\Omega^{\prime}$ is the scattered electron solid angle, $\sigma_{M}$ is the Mott cross section $[4,5]$ and

$$
F_{p}(q)=\int \mathrm{d} \mathbf{r} \jmath_{0}(q r) \rho_{p}(r),
$$

is the charge form factor for a spherical nuclear charge (point proton) density $\rho_{p}(r)$ and $\jmath_{0}$ is the zeroth order spherical Bessel function.

The PWBA is, however, not adequate for medium and heavy nuclei where the distortion produced on the electron wave functions by the nuclear Coulomb potential $V(r)$ from $\rho_{p}(r)$ can have significant effects. The DWBA cross sections are obtained from the numerical solutions of the partial wave Dirac equation.

\section{B. Inclusive quasi-elastic electron scattering}

In the one-photon exchange approximation the inclusive differential cross section for the $\mathrm{QE}\left(e, e^{\prime}\right)$ scattering on a nucleus is obtained from the contraction between the lepton and hadron tensors as $[5]$

$$
\left(\frac{\mathrm{d} \sigma}{\mathrm{d} \varepsilon^{\prime} \mathrm{d} \Omega^{\prime}}\right)_{Q E}=\sigma_{M}\left[v_{L} R_{L}+v_{T} R_{T}\right],
$$

where $\varepsilon^{\prime}$ is the energy of the scattered electron. The coefficients $v$ come from the components of the lepton tensor that, under the assumption of the plane-wave approximation for the electron wave functions, depend only on the lepton kinematics,

$$
v_{L}=\left(\frac{\left|Q^{2}\right|}{|\boldsymbol{q}|^{2}}\right)^{2}, v_{T}=\tan ^{2} \frac{\theta}{2}-\frac{\left|Q^{2}\right|}{2|\boldsymbol{q}|^{2}},
$$


where $\theta$ is the electron scattering angle and $Q^{2}=|\boldsymbol{q}|^{2}-\omega^{2}$. All nuclear structure information is contained in the longitudinal and transverse response functions $R_{L}$ and $R_{T}$, expressed by

$$
\begin{aligned}
& R_{L}(q, \omega)=W^{00}(q, \omega), \\
& R_{T}(q, \omega)=W^{11}(q, \omega)+W^{22}(q, \omega)
\end{aligned}
$$

in terms of the diagonal components of the hadron tensor, that is given by bilinear products of the transition matrix elements of the nuclear electromagnetic many-body current operator $\hat{J}^{\mu}$ between the initial state of the nucleus $\left|\Psi_{0}\right\rangle$, of energy $E_{0}$, and the final states $\left|\Psi_{f}\right\rangle$, of energy $E_{f}$, both eigenstates of the nuclear Hamiltonian $H$, as

$$
\begin{aligned}
W^{\mu \mu}(q, \omega) & =\overline{\sum_{i}} \oint_{f}\left\langle\Psi_{f}\left|\hat{J}^{\mu}(\boldsymbol{q})\right| \Psi_{0}\right\rangle \\
& \times\left\langle\Psi_{0}\left|\hat{J}^{\mu \dagger}(\boldsymbol{q})\right| \Psi_{f}\right\rangle \delta\left(E_{0}+\omega-E_{f}\right),
\end{aligned}
$$

involving an average over the initial states and a sum over the undetected final states. The sum runs over the scattering states corresponding to all of the allowed asymptotic configurations and includes possible discrete states $[4,5]$.

The hadron tensor can equivalently be expressed as

$$
W^{\mu \mu}(q, \omega)=-\frac{1}{\pi} \operatorname{Im}\left\langle\Psi_{0}\left|J^{\mu \dagger}(\boldsymbol{q}) G\left(E_{f}\right) J^{\mu}(\boldsymbol{q})\right| \Psi_{0}\right\rangle
$$

where $E_{f}=E_{0}+\omega$ and $G\left(E_{f}\right)$ is the many-body Green's function related to the many-body nuclear Hamiltonian $H$.

The hadron in tensor in Eq. (7) contains the full many-body propagator of the nuclear system. As such, it is an extremely complicated object and some approximations are needed to reduce the calculation of the nuclear response to a tractable form.

\section{Parity-violating electron scattering}

When a photon is exchanged between two charged particles a $Z^{0}$ boson is also exchanged. At the energies of interest in electron scattering the strength of the weak process mediated by the $Z^{0}$ boson is negligible compared with the electromagnetic strength. The role played by the $Z^{0}$ exchange is therefore not significant unless an experiment is set up to measure a parity-violating observable. While the electromagnetic interaction conserves parity, the weak interaction does not and this is how we are sensitive to $Z^{0}$ exchange in electron scattering. 
The degree of parity violation can be measured by the parity-violating asymmetry $A_{p v}$, or helicity asymmetry, which is defined as the difference between the cross sections for the scattering of electrons longitudinally polarized parallel and antiparallel to their momentum. This difference arises from the interference of photon and $Z^{0}$ exchange. As it has been shown in Refs. [49, 60], the asymmetry in the parity violating elastic polarized electron scattering represents an almost direct measurement of the Fourier transform of the neutron density.

The electron spinor for elastic scattering on a spin-zero nucleus can be written as the solution of a Dirac equation with total potential

$$
U(r)=V(r)+\gamma_{5} A(r)
$$

where $V(r)$ is the Coulomb potential and $A(r)$ is the axial potential which results from the weak neutral current amplitude and which depends on the Fermi constant $G_{F} \simeq 1.16639 \times$ $10^{-11} \mathrm{MeV}^{-2}$, i.e.,

$$
A(r)=\frac{G_{F}}{2 \sqrt{2}} \rho_{W}(r),
$$

The weak charge density $\rho_{W}$ is related to the neutron density and it is defined

$$
\begin{aligned}
\rho_{W}(r)= & \int \mathrm{d} \mathbf{r}^{\prime} G_{E}\left(\left|\mathbf{r}-\mathbf{r}^{\prime}\right|\right) \\
& \times\left[-\rho_{n}\left(r^{\prime}\right)+\left(1-4 \sin ^{2} \Theta_{W}\right) \rho_{p}\left(r^{\prime}\right)\right],
\end{aligned}
$$

where $\rho_{n}$ and $\rho_{p}$ are point neutron and proton densities, $G_{E}(r) \approx \frac{\Lambda^{3}}{8 \pi} e^{-\Lambda r}$ is the electric form factor of the proton, with $\Lambda=4.27 \mathrm{fm}^{-1}$, and $\sin ^{2} \Theta_{W} \simeq 0.23$ is the Weinberg angle. The axial potential of Eq. (9) is much smaller than the vector potential and, since $1-4 \sin ^{2} \Theta_{W} \ll$ 1 , it depends mainly on the neutron distribution $\rho_{n}(r)$.

In the limit of vanishing electron mass, the helicity states $\Psi_{ \pm}=\frac{1}{2}\left(1 \pm \gamma_{5}\right) \Psi$ satisfy the Dirac equation

$$
\left[\boldsymbol{\alpha} \cdot \mathbf{p}+U_{ \pm}(r)\right] \Psi_{ \pm}=E \Psi_{ \pm}
$$

with

$$
U_{ \pm}(r)=V(r) \pm A(r) .
$$

The parity-violating asymmetry $A_{p v}$, or helicity asymmetry, is defined

$$
A_{p v}=\frac{\frac{d \sigma_{+}}{d \Omega}-\frac{d \sigma_{-}}{d \Omega}}{\frac{d \sigma_{+}}{d \Omega}+\frac{d \sigma_{-}}{d \Omega}}
$$


where $+(-)$ refers to the elastic scattering on the potential $U_{ \pm}(r)$. In Born approximation, neglecting strangeness contributions and the electric neutron form factor, the parity-violating asymmetry can be rewritten as $[61,79]$

$$
A_{p v}=\frac{G_{F} Q^{2}}{4 \sqrt{2} \pi \alpha}\left[4 \sin ^{2} \Theta_{W}-1+\frac{F_{n}(q)}{F_{p}(q)}\right] .
$$

Since $4 \sin ^{2} \Theta_{W}-1$ is small and $F_{p}(q)$ is known, we see that $A_{p v}$ provides a practical method to measure the neutron form factor $F_{n}(q)$ and hence the neutron radius. For these reasons parity-violating electron scattering (PVES) has been suggested as a clean and powerful tool for measuring the spatial distribution of neutrons in nuclei.

In QE electron scattering the helicity asymmetry is obtained in terms of kinematic coefficients $v$ from the lepton tensor and of nuclear response functions as [72]

$$
A_{p v}^{Q E}=A_{0} \frac{v_{L} R_{L}^{A V}+v_{T} R_{T}^{A V}+v_{T}^{\prime} R_{T}^{V A}}{v_{L} R_{L}+v_{T} R_{T}} .
$$

The factor $A_{0}$ is defined as

$$
A_{0}=\frac{G_{F} Q^{2}}{2 \sqrt{2} \pi \alpha},
$$

where $\alpha$ is the fine structure constant. The denominator in Eq. (15) contains the parityconserving cross section of Eq. (3), the numerator the parity-violating contribution,

$$
v_{T}^{\prime}=\tan \frac{\theta}{2} \sqrt{\tan ^{2} \frac{\theta}{2}+\frac{Q^{2}}{|\boldsymbol{q}|^{2}}}
$$

and the response functions $R$ are given in terms of the polarized components of the hadron tensor $W_{I}^{\mu \nu}[72]$ as

$$
\begin{aligned}
& R_{L}^{A V}=g_{A} W_{I}^{00}, R_{T}^{A V}=g_{A}\left(W_{I}^{11}+W_{I}^{22}\right), \\
& R_{T}^{V A}=i g_{V}\left(W_{I}^{12}-W_{I}^{21}\right),
\end{aligned}
$$

where the superscript AV denotes interference of axial-vector leptonic current with vector

hadronic current (the reverse for VA) and the couplings $g_{A}=-\frac{1}{2}$ and $g_{V}=-\frac{1}{2}+2 \sin ^{2} \Theta_{W} \simeq$ -0.04 .

\section{RELATIVISTIC MODEL FOR GROUND-STATE OBSERVABLES}

In the standard representation of relativistic mean field approaches the nucleus is described as a system of Dirac nucleons coupled to the exchange mesons and the electromagnetic field through an effective Lagrangian. The isoscalar scalar-meson $(\sigma)$, the isoscalar 
vector-meson $(\omega)$, and the isovector vector-meson $(\rho)$ build the minimal set of meson fields that together with the electromagnetic field $(\gamma)$ is necessary for a quantitative description of bulk and s.p. nuclear properties. The model is defined by the Lagrangian density

$$
\mathcal{L}=\mathcal{L}_{N}+\mathcal{L}_{m}+\mathcal{L}_{\text {int }}
$$

where $\mathcal{L}_{N}$ denotes the Lagrangian of the free nucleon, $\mathcal{L}_{m}$ is the Lagrangian of the free meson fields and the simplest set of interaction terms is contained in $\mathcal{L}_{\text {int }}$ :

$$
\mathcal{L}_{i n t}=-g_{\sigma} \bar{\psi} \sigma \psi-g_{\omega} \bar{\psi} \gamma^{\mu} \omega_{\mu} \psi-g_{\rho} \bar{\psi} \gamma^{\mu} \vec{\tau} \cdot \vec{\rho}_{\mu} \psi
$$

The couplings of the $\sigma$-meson and $\omega$-meson to the nucleon are assumed to be of the form:

$$
g_{i}(\rho)=g_{i}\left(\rho_{\text {sat }}\right) f_{i}(x) \text { for } \quad i=\sigma, \omega,
$$

where

$$
f_{i}(x)=a_{i} \frac{1+b_{i}\left(x+d_{i}\right)^{2}}{1+c_{i}\left(x+d_{i}\right)^{2}}
$$

is a function of $x=\rho / \rho_{\text {sat }}$ and $\rho_{\text {sat }}=0.152 \mathrm{fm}^{-3}$ denotes the nucleon density at saturation in symmetric nuclear matter. Constraints at nuclear matter saturation density and at zero density are used to reduce the number of independent parameters in Eq. (22) to three. Three additional parameters in the isoscalar channel are $g_{\sigma}\left(\rho_{\text {sat }}\right), g_{\omega}\left(\rho_{\text {sat }}\right)$, and $m_{\sigma}$, that is the mass of the phenomenological $\sigma$ meson. For the $\rho$ meson coupling the functional form of the density dependence is suggested by Dirac-Brueckner calculations of asymmetric nuclear matter:

$$
g_{\rho}(\rho)=g_{\rho}\left(\rho_{\text {sat }}\right) \exp \left[-a_{\rho}(x-1)\right]
$$

and the isovector channel is parametrized by $g_{\rho}\left(\rho_{\text {sat }}\right)$ and $a_{\rho}$. Bare values are used for the masses of the $\omega$ and $\rho$ mesons: $m_{\omega}=783 \mathrm{MeV}$ and $m_{\rho}=763 \mathrm{MeV}$. DD-ME2 is determined by eight independent parameters, adjusted to the properties of symmetric and asymmetric nuclear matter, binding energies, charge radii, and neutron radii of spherical nuclei [68]. The interaction has been tested in the calculation of ground state properties of a large set of spherical and deformed nuclei. When used in the relativistic random-phase approximation, DD-ME2 reproduces with high accuracy data on isoscalar and isovector collective excitations. For open-shell nuclei we employed a schematic ansatz: the constant gap approximation with 
empirical $\Delta$ given by the 5 -point formula [80]

$$
\begin{aligned}
\Delta^{(5)}\left(N_{0}\right)=-\frac{1}{8} & {\left[E\left(N_{0}+2\right)-4 E\left(N_{0}+1\right)+6 E\left(N_{0}\right)\right.} \\
& \left.-4 E\left(N_{0}-1\right)+E\left(N_{0}-2\right)\right] .
\end{aligned}
$$

In Figs. 1 and 2 we plot the neutron (proton) density distributions $\rho_{n(p)}$ as a function of the radial coordinate $r$ for oxygen and calcium isotopes, respectively. These density distributions are the sum of the squared moduli of the s.p. neutron (proton) wave functions. All the nuclei we have investigated resulted to be bound. From the experimental point of view it seems rather well established that the neutron drip line for the oxygen isotopes starts with ${ }^{26} \mathrm{O}$ [81] and, therefore, ${ }^{28} \mathrm{O}$ should not be bound.

For both isotopic chains the differences between the proton and neutron densities in Figs. 1 and 2 generally increase with the neutron number. When the number of neutrons increases there is a gradual increase of the neutron radius. The differences of the neutron density profiles in the nuclear interior display pronounced shell effects. The effect of adding neutrons is to populate and extend the neutron densities and, to a minor extent, also the proton densities. In the case of protons, however, there is a decrease of the density in the nuclear interior to preserve the normalization to the constant number of protons.

\section{RELATIVISTIC GREEN'S FUNCTION MODEL FOR INCLUSIVE QUASI-ELASTIC ELECTRON SCATTERING}

In the QE region the nuclear response is dominated by one-nucleon knockout processes, where the scattering occurs with only one nucleon that is subsequently emitted. The remaining nucleons of the target behave as simple spectators and QE electron scattering can adequately be described in the relativistic impulse approximation (RIA) by the sum of incoherent processes involving only one nucleon scattering and the components of the hadron tensor of Eq. (6) are obtained from the sum, over all the s.p. shell-model states, of the squared absolute value of the transition matrix elements of the single-nucleon current.

A reliable description of final-state interactions (FSI) between the ejected nucleon and the residual nucleus is an essential ingredient for the comparison with data. In the case of exclusive $\left(e, e^{\prime} p\right)$ processes, the use of complex optical potentials in the relativistic distorted wave impulse approximation (RDWIA) has been able to successfully describe a wide number 
of experimental data $[16,47,48,82-86]$. It is clear that the pure RDWIA approach, based on the use of an absorptive complex potential, would be inconsistent in the analysis of inclusive scattering, where all final-state channels should be retained and the total flux, although redistributed among all possible channels due to FSI, must be conserved. Different approaches have been used to describe FSI in RIA calculations for the inclusive QE electronand neutrino-nucleus scattering [70-75, 77, 87-96]. In the relativistic plane-wave impulse approximation (RPWIA), FSI are simply neglected. In another approach, FSI are included in calculations where the final nucleon state is evaluated with real potentials, either retaining only the real part of the relativistic energy-dependent complex optical potential (rROP) [95, 96], or using the same relativistic mean field potential considered in describing the initial nucleon state [91].

In the relativistic Green's function (RGF) model FSI are described in the inclusive process consistently with the exclusive scattering by the same complex optical potential, but the imaginary part is used in the two cases in a different way and in the inclusive scattering it redistributes the flux in all the channels and the total flux is conserved. Detailed discussions of the RGF model can be found in Refs. [70-77, 97, 98]. The model assumes that the ground state of the nucleus $\left|\Psi_{0}\right\rangle$ is non-degenerate, this is a suitable approximation for the even isotopes of oxygen and calcium considered in this work, with spin and parity $0^{+}$[99].

In the RGF model with suitable approximations, which are mainly related to the impulse approximation, the components of the nuclear response of Eq. (7) are written in terms of the s.p. optical model Green's function. The spectral representation of the s.p. Green's function, which is based on a biorthogonal expansion in terms of a non-Hermitian optical potential and of its Hermitian conjugate, can be exploited to avoid the explicit calculation of the s.p. Green's function and obtain the components of the hadron tensor in the form $[70]$

$$
\begin{gathered}
W^{\mu \mu}(q, \omega)=\sum_{n}\left[\operatorname{Re} T_{n}^{\mu \mu}\left(E_{f}-\varepsilon_{n}, E_{f}-\varepsilon_{n}\right)\right. \\
\left.-\frac{1}{\pi} \mathcal{P} \int_{M}^{\infty} \mathrm{d} \mathcal{E} \frac{1}{E_{f}-\varepsilon_{n}-\mathcal{E}} \operatorname{Im} T_{n}^{\mu \mu}\left(\mathcal{E}, E_{f}-\varepsilon_{n}\right)\right],
\end{gathered}
$$

where $\mathcal{P}$ denotes the principal value of the integral, $n$ is the eigenstate of the residual nucleus 
with energy $\varepsilon_{n}$, and

$$
\begin{aligned}
T_{n}^{\mu \mu}(\mathcal{E}, E)= & \lambda_{n}\left\langle\varphi_{n}\left|j^{\mu \dagger}(\boldsymbol{q}) \sqrt{1-\mathcal{V}^{\prime}(E)}\right| \tilde{\chi}_{\mathcal{E}}^{(-)}(E)\right\rangle \\
& \times\left\langle\chi_{\mathcal{E}}^{(-)}(E)\left|\sqrt{1-\mathcal{V}^{\prime}(E)} j^{\mu}(\boldsymbol{q})\right| \varphi_{n}\right\rangle .
\end{aligned}
$$

The factor $\sqrt{1-\mathcal{V}^{\prime}(E)}$, where $\mathcal{V}^{\prime}(E)$ is the energy derivative of the optical potential, accounts for interference effects between different channels and justifies the replacement in the calculations of the Feshbach optical potential $\mathcal{V}$ of the RGF model by the local phenomenological optical potential [70, 97, 98].

Disregarding the square root correction, the second matrix element in Eq. (26) is the transition amplitude of the usual RDWIA model for the exclusive single-nucleon knockout. In this matrix element $j^{\mu}$ is the one-body nuclear current, $\chi^{(-)}$is the s.p. scattering state of the emitted nucleon with energy $\mathcal{E}, \varphi_{n}$ is the overlap between the ground state of the target and the final state $n$, i.e., a s.p. bound state, and the spectroscopic factor $\lambda_{n}$ is the norm of the overlap function. In the model $\varphi_{n}$ and $\chi^{(-)}$are consistenlty derived as eigenfunctions of the energy-dependent optical-model Hamiltonian at bound and scattering energies.

In the exclusive one-nucleon knockout the imaginary part of the optical potential accounts for the flux lost in the channel $n$ towards the channels different from $n$, which are not included in the exclusive process. In the inclusive response, where all the channels are included, this loss is compensated by a corresponding gain of flux due to the flux lost, towards the channel $n$, in the other final states asymptotically originated by the channels different from n. This compensation is performed by the first matrix element in the right hand side of Eq. (26), which involves the eigenfunction $\tilde{\chi}_{\mathcal{E}}^{(-)}(E)$ of the Hermitian conjugate optical potential, where the imaginary part has an opposite sign and has the effect of increasing the strength. Therefore, in the RGF approach the imaginary part of the optical potential redistributes the flux lost in a channel in the other channels, and in the sum over $n$ the total flux is conserved. If the imaginary part of the optical potential is neglected, the second term in Eq. (25) vanishes and, but for the square root factor, the first term gives the rROP approach.

In Eq. (26) $\tilde{\chi}^{(-)}$and $\chi^{(-)}$are therefore eigenfunctions of the optical potential $\mathcal{V}(E)$ and of its Hermitian conjugate $\mathcal{V}^{\dagger}(E)$, which are nonlocal operators with a possibly complicated matrix structure. Neither microscopic nor empirical calculations of $\mathcal{V}(E)$ are available. Only phenomenological local optical potentials, obtained through fits to elastic nucleon-nucleus 
scattering data, are available. These phenomenological optical potentials are used in RGF calculations. As no relativistic optical potentials are available for the bound states, the overlap functions $\varphi_{n}$, are computed in the present work using the model discussed in Sect. III.

The RGF model has been applied to parity-violating QE electron scattering in Ref. [72]. The main steps of the model are the same, the expressions for the electromagnetic-weak interference components of the hadron tensor $W_{I}^{\mu \nu}$ can be found in Ref. [72].

\section{RESULTS}

In this section we present and discuss numerical predictions for elastic and QE electron scattering which can hopefully be useful for future measurements in experimental RIB facilities. We study the evolution of some electron scattering observables in isotopic chains of medium systems, which are exemplified by the cases of the oxygen and calcium isotopes. Many of these nuclei lie in the region of the nuclear chart that is likely to be explored in future electron-scattering experiments. As a first step, with a few numerical examples we test the results of our models in comparison with available data. Then, for each nucleus in an isotopic chain, we compute and compare the associated elastic and QE cross sections and parity-violating asymmetry in order to obtain information on the effects of isospin asymmetry on nuclear structure.

\section{A. Elastic electron scattering}

The cross sections for elastic electron scattering have been calculated in the DWBA and with the self-consistent relativistic ground state charge densities described in Sect. III. In the PWBA the cross section is proportional to the Fourier transform of the proton charge density (see Eq. (2)) and reflects its behavior also when Coulomb distortion is included in the calculations. In different studies of the charge form factors along isotopic chains $[36,40,41,100,101]$ it has been found that, when the number of neutrons increases, the squared modulus of the charge form factor and the position of its minima show, respectively, an upward trend and a significant inward shifting in the momentum transfer.

An example of the comparison between theoretical and experimental differential cross 
sections is displayed in Fig. 3 for elastic electron scattering on ${ }^{16} \mathrm{O}$ at an electron energy $\varepsilon=374.5 \mathrm{MeV}$ and on ${ }^{40} \mathrm{Ca}$ at $\varepsilon=496.8 \mathrm{MeV}$. The general trend of the experimental data is reasonably reproduced by the calculations. Both experimental cross sections considered in the figure are well described at low scattering angles. For ${ }^{40} \mathrm{Ca}$ there is a fair agreement between theory and data also at larger angles, while for ${ }^{16} \mathrm{O}$ data beyond the minimum are somewhat underestimated by the theoretical results.

The calculated differential cross sections for elastic electron scattering on various oxygen isotopes $\left({ }^{14-28} \mathrm{O}\right)$ at $\varepsilon=374.5 \mathrm{MeV}$ and on calcium isotopes $\left({ }^{36-56} \mathrm{Ca}\right)$ at $\varepsilon=496.8 .5 \mathrm{MeV}$ are shown in Figs. 4 and 5, respectively. With increasing neutron number the positions of the diffraction minima shift toward smaller scattering angles, i.e., towards smaller values of the momentum transfer. The shift of the minima towards smaller $q$ is in general accompanied by a simultaneous increase in the height of the maxima. The behavior is similar for both isotopic chains here considered and is in agreement with the results found in previous studies of charge form factors on various isotopic chains, which were carried out with different meanfield models [36, 40, 41, 100, 101].

\section{B. Quasi-elastic electron scattering}

The cross sections for QE electron scattering have been computed with the RGF model discussed in Sect. IV. Some results obtained in the RPWIA are also presented for a comparison. In the calculations of the matrix elements in Eq. (26) the s.p. bound nucleon states are obtained from the relativistic mean-field model with density-dependent meson-nucleon vertices and the DD-ME2 parametrization as described in Sect. III. The s.p. scattering states are eigenfunctions of the energy-dependent and $A$-dependent ( $A$ is the mass number) parameterization for the relativistic optical potential of Ref. [102], which is fitted to proton elastic scattering data on several nuclei in an energy range up to $1040 \mathrm{MeV}$. The different number of neutrons along the $\mathrm{O}$ and $\mathrm{Ca}$ isotopic chains produces different optical potentials (see Ref. [102] for more details). For the single-nucleon current we have used the relativistic free nucleon expression denoted as CC2 [70, 103].

The predictions of the RGF model have been compared with experimental data for QE electron- and neutrino-nucleus scattering in a series of papers [70-77, 89, 93], where the calculations have been performed with different relativistic mean-field models for the bound 
states and different parameterizations of the relativistic optical potential.

In Fig. 6 our RGF results are compared with the experimental $\left(e, e^{\prime}\right)$ cross sections for two different kinematics on ${ }^{16} \mathrm{O}$ and ${ }^{40} \mathrm{Ca}$ target nuclei $[104,105]$. The agreement with the data is satisfactory, at least in the energy region of the QE peak. The RGF model was developed to describe FSI in inclusive QE electron scattering and is in general able to give a reasonable and even good description of QE data. For energy regions below and above the QE peak other contributions, not included in the RGF model, can be important. Even in the QE region, the relevance of contributions like meson exchange currents and Delta effects should be carefully evaluated before definite conclusions can be drawn about the comparison with data [106-108]. Such contributions may be significant even in the QE region, in particular in kinematics where the transverse component of the nuclear response plays a major role in the cross section.

The cross section of the inclusive $\mathrm{QE}\left(e, e^{\prime}\right)$ reaction on ${ }^{14-28} \mathrm{O}$ isotopes at $\varepsilon=1080 \mathrm{MeV}$ and $\theta=32^{\circ}$ are shown in Fig. 7. In a first approximation, we have neglected FSI and calculations have been performed in the RPWIA. In this case, the differences between the results for the various isotopes are entirely due to the differences in the s.p. bound state wave functions of each isotope. While only the charge proton density distribution contributes to the cross section of elastic electron scattering, the cross section of QE electron scattering is obtained from the sum of all the integrated exclusive one-nucleon knockout processes, due to the interaction of the probe with all the individual nucleons, protons and neutrons, of the nucleus and contains information on the dynamics of the initial nuclear ground state. The separate contributions from protons and neutrons are also shown in the lower panel of Fig. 7. In an usual experiment where only the scattered electron is detected these two quantities cannot be separated experimentally, but their comparison can give useful information on the different role of protons and neutrons in the inclusive QE cross section. The main role is played by protons, which give most of the contribution. Increasing the neutron number it is quite natural to understand the proportional increase of the neutron contribution. No significant increase is found in the proton contribution. Thus, the increase of the cross section in the upper panel of the figure is due to the increase of the neutron contribution. The shift of the proton contribution towards higher values of $\omega$ seen in the figure is mainly related to the increase of the proton separation energy with increasing neutron number (increasing the neutron number the protons experience more binding and their separation 
energies increase) than to changes in the proton wave functions. A different and opposite shift can be seen in the case of the neutron contribution and, therefore, the final effect is that the shift is strongly reduced in the QE cross section shown in the upper panel of Fig. 7.

In Fig. 8 we show the $\mathrm{QE}\left(e, e^{\prime}\right)$ cross sections calculated for oxygen isotopes with the RGF model and in the same kinematics as in Fig. 7. The general trend of the cross sections, their magnitude, and their evolution with respect to the change of the neutron number are generally similar in RPWIA and RGF. The FSI effects in the RGF calculations produce, however, some differences which can be seen in the low energy transferred region, where the cross sections for ${ }^{14,16,18} \mathrm{O}$ are enhanced with respect to those for ${ }^{22} \mathrm{O}$ and ${ }^{28} \mathrm{O}$. In addition, the shift towards higher $\omega$ is more significant than in the RPWIA case, but for ${ }^{28} \mathrm{O}$.

The cross section of the inclusive $\mathrm{QE}\left(e, e^{\prime}\right)$ reaction on ${ }^{36-56} \mathrm{Ca}$ isotopes at $\varepsilon=560$ $\mathrm{MeV}$ and $\theta=60^{\circ}$ calculated in the RPWIA and in the RGF are shown in Figs. 9 and 10, respectively. The general behavior of the cross sections and their evolution with increasing neutron number is similar for calcium and oxygen isotopes. The magnitude increases with the neutron number, but FSI effects are somewhat more visible for calcium isotopes. The RGF cross sections on ${ }^{36,40,44,48} \mathrm{Ca}$ in Fig. 10 are enlarged over a wide range of $\omega$ and are slightly reduced with respect to the RPWIA results in Fig. 9. This is particularly visible for ${ }^{48} \mathrm{Ca}$ and produces an apparently large gap between the cross sections of ${ }^{48} \mathrm{Ca}$ and ${ }^{52} \mathrm{Ca}$.

As a final comment, we can add that interesting and peculiar effects are obtained in the evolution of QE inclusive cross section along isotopic chains, but it is not easy to relate them to changes in the matter distribution, which can be significant, particularly in the center of the nucleus.

\section{Parity-violating asymmetry}

The calculation starts with the self-consistent relativistic ground state proton and neutron densities (see Sect. III). The charge and weak densities are calculated by folding the point proton and neutron densities (see Eq. (10)). The resulting Coulomb potential $V(r)$ and weak potential $A(r)$ (see Eq.(9)) are used to construct $U_{ \pm}(r)$. The cross sections for elastic electron scattering are obtained from the numerical solution of the Dirac equation for electron scattering in the $U_{ \pm}(r)$ potential and includes Coulomb distortion effects [36, 51, 56, 109]. The cross sections for positive and negative helicity electron states are calculated and the 
resulting asymmetry parameter $A_{p v}$ is plotted as a function of the scattering angle.

In Figs. 11 and 12 we plot the parity-violating asymmetry parameters $A_{p v}$ for ${ }^{14-28} \mathrm{O}$ and ${ }^{36-56} \mathrm{Ca}$ nuclei for elastic electron scattering at $\varepsilon=850 \mathrm{MeV}$. At $\varepsilon=850 \mathrm{MeV}$ the values of $A_{p v}$ are of the order of $10^{-5}$, with lower values for smaller angles and larger values for larger angles.

As suggested in Ref. [49] the asymmetry parameter $A_{p v}$ provides a direct measurement of the Fourier transform of the neutron density. This relation has been tested and confirmed in Ref. [60] comparing asymmetries and the squares of the Fourier transforms of the neutron densities. Another way to relate $A_{p v}$ to neutron distributions of finite nuclei is by looking at possible linear correlations between the asymmetry parameter and some well defined observables. We suggest to use the first minima positions $\theta_{\min }$ and the neutron excess $\Delta=\frac{N-Z}{Z}$, i.e., how the minima of $A_{p v}$ evolve from neutron-poor to neutron-rich nuclei (see Eqs. (13) and (14)). In Fig. 13, $\theta_{\min }$ is plotted as a function of $\Delta$ for oxygen and calcium isotopes. The dashed lines suggest that for both isotope chains the evolution of $A_{p v}$ as function of $\Delta$ is well approximated by a linear fit with a very similar slope. To test the robustness of this correlation it is interesting to study if $A_{p v}$ is affected by density distribution oscillations at small radii that could appear in some selected cases. In Ref. [39], ${ }^{22} \mathrm{O}$ and ${ }^{24} \mathrm{O}$ isotopes have been studied as possible candidates for "bubble" nuclei, i.e., nuclear systems with a strong depleted central density. In Fig. 14 we plot the asymmetry parameters $A_{p v}$ for these nuclei. Neutron density profiles show large differences at small distances. No appreciable effects are obtained in the corresponding asymmetries up to $\theta \simeq 20^{\circ}$, then for larger scattering angles the asymmetries are sensitive to the differences in the density distributions and are significantly different. Therefore, $A_{p v}$ is still a reliable observable to study neutron radii even if we include pairing correlations, but we must limit to angles smaller than the first minimum position.

In addition to predictions about oxygen and calcium isotopic chains we also provide calculations for recent measurements and future experiments. In Fig. 15 we show our theoretical predictions for the empirical values extracted from the first run of the PREX experiment on ${ }^{208} \mathrm{~Pb}$ at $\varepsilon=1.06 \mathrm{GeV}$. In Ref. [53] the weak charge density $\left(-\rho_{W}\right)$ has been deduced from the weak charge form factor. The error band (shaded area) represents the incoherent sum of experimental and model errors. Our prediction, plotted by the red line in the left panel, is in rather good agreement with empirical data. In fact, if we evaluate the 
corresponding asymmetry parameter $A_{p v}$ averaged over the acceptance function $\epsilon(\theta)[110]$

$$
\left\langle A_{p v}\right\rangle=\frac{\int \mathrm{d} \theta \sin \theta A_{p v}(\theta) \frac{d \sigma}{d \Omega} \epsilon(\theta)}{\int \mathrm{d} \theta \sin \theta \frac{d \sigma}{d \Omega} \epsilon(\theta)}
$$

we find $0.712 \mathrm{ppm}$, in very good agreement with the empirical estimate $0.656 \pm 0.060$ (stat) \pm 0.014 (syst) ppm. In Fig. 16 we calculate the asymmetry parameter for ${ }^{48} \mathrm{Ca}$ with 2.2 $\mathrm{GeV}$ electrons as planned for the CREX experiment. For energies well above the $1 \mathrm{GeV}$ region, of course, the elastic scattering approximation is not completely under control and corrections due to possible inelasticities should be taken into account. We plan to extend our calculation in a forthcoming paper.

As an example of the parity-violating asymmetry for QE scattering, in Fig. 17 we show $A_{p v}$ for the ${ }^{14,16,18,22,28} \mathrm{O}$ isotopes evaluated with the RGF in the same kinematics as in Fig. 8. Note that the results are rescaled by the factor $10^{5}$. The RPWIA results are always similar to the RGF ones and are not presented here. The asymmetry is almost constant to a few $\times 10^{-6}$ for ${ }^{14} \mathrm{O}$ and ${ }^{16} \mathrm{O}$, whereas for ${ }^{22} \mathrm{O}$ and ${ }^{28} \mathrm{O}$ it goes up to $\approx-2 \times 10^{-5}$ in the low energy transferred region. There are visible relative differences between the asymmetries for the oxygen isotopes that we have considered, but we are aware that a measurement of $A_{p v}$ in QE electron scattering off finite nuclei is extremely challenging with the presently available facilities.

\section{SUMMARY AND CONCLUSIONS}

We have presented and discussed numerical predictions for the cross section and the parity-violating asymmetry in elastic and quasi-elastic electron scattering on oxygen and calcium isotope chains with the aim to investigate their evolution with increasing neutron number.

The understanding of the properties of exotic nuclei is one of the major topic of interest in modern nuclear physics. Large efforts in this directions have been done over last years and are planned for the future. The use of electrons as probe provides a powerful tool to achieve this goal. The RIB facilities in different laboratories have opened the possibility to give insight into nuclear structures which are not available in nature, as they are not stable, but which are important in astrophysics and had a relevant role in the nucleosynthesis. 
Electron scattering is well fitted for studying nuclear properties, as its interaction is well known and relatively weak with respect to the hadron force and can therefore more adequately explore the details of inner nuclear structures. As a consequence of this weakness, the cross sections become very small and more difficult experiments have to be performed. Electron scattering experiments off exotic nuclei have been proposed in the ELISe experiment at FAIR and in the SCRIT project at RIKEN. We hope that the existing proposals will be considered and approved in next years. Our theoretical predictions will be useful for clarifying the different aspects of the measurements, giving information on the order of magnitude of the measurable quantities and therefore making possible a more precise evaluation of the experimental difficulties. Moreover, a theoretical investigation can be helpful to envisage the most interesting quantities to be measured in order to explore the properties of exotic nuclear structures.

In this work, both elastic and inclusive quasi-elastic electron scattering have been considered. The elastic scattering can give information on the global properties of nuclei and, in particular, on the different behavior of proton and neutron density distributions. The inclusive quasi-elastic scattering is affected by the dynamical properties, being the integral of the spectral density function over all the available final states, and, due to the reaction mechanism, preferably exploits the single particle aspects of the nucleus. In addition, when combined with the exclusive $\left(e, e^{\prime} p\right)$ scattering, it is able to explore the evolution of the single particle model with increasing asymmetry between the number of neutrons and protons. Many interesting phenomena are predicted in this situation: in particular, the modification of the shell model magic numbers. A definite response can be obtained from the comparison with experimental data, which will discriminate between the different theoretical models, mainly referring to relativistic mean field approaches.

As case studies for the present investigation we have selected oxygen and calcium isotope chains. The calculations have been carried out within the framework of the relativistic mean field model. The nuclear wave functions are obtained considering a system of nucleons coupled to the exchange mesons and the electromagnetic field through an effective Lagrangian. The calculated cross sections include both the hadronic and Coulomb final states interactions. The inclusive quasi-elastic scattering is calculated with the relativistic Green's function model, which conserves the global particle flux in all the final state channels, as it is required in an inclusive reaction. 
First, the models have been compared with experimental data already available on stable isotopes in order to check their reliability. Then, the same models have been used to calculate elastic and inclusive quasi-elastic cross sections on exotic isotopes chains. The possible disagreement of the experimental findings from the theoretical predictions will be a clear indication of the insurgence of new phenomena related to the proton to neutron asymmetry.

Our results show an evolution of the calculated quantities without discontinuities. The increase of the neutron number essentially produces an increase of the nuclear and proton densities and a flattening of the charge density.

The parity-violating asymmetry parameter has been calculated in order to investigate the neutron skin, as the weak current is essentially obtained from the interaction with neutrons. Numerical predictions have been presented and discussed for oxygen and calcium isotopes. Moreover, we have compared our calculations with the results of the first measurement of the asymmetry parameter achieved by the PREX Collaboration on ${ }^{208} \mathrm{~Pb}$ and have obtained a good agreement with the empirical value. A prediction for the future experiment CREX on ${ }^{48} \mathrm{Ca}$ has also been given.

\section{ACKNOWLEDGMENTS}

This work was partially supported by the Italian MIUR through the PRIN 2009 research project.

[1] R. Hofstadter, Rev. Mod. Phys. 28, 214 (1956).

[2] T. W. Donnelly and J. D. Walecka, Ann. Rev. Nucl. Part. Sci. 25, 329 (1975).

[3] T. W. Donnelly and I. Sick, Rev. Mod. Phys. 56, 461 (1984).

[4] S. Boffi, C. Giusti, and F. D. Pacati, Phys. Rept. 226, 1 (1993).

[5] S. Boffi, C. Giusti, F. D. Pacati, and M. Radici, Electromagnetic Response of Atomic Nuclei, Oxford Studies in Nuclear Physics, Vol. 20 (Clarendon Press, Oxford, 1996).

[6] O. Benhar, D. Day, and I. Sick, Rev. Mod. Phys. 80, 189 (2008).

[7] H. De Vries, C. W. De Jager, and C. De Vries, Atom. Data Nucl. Data Tabl. 36, 495 (1987).

[8] G. Fricke, C. Bernhardt, K. Heilig, L. A. Schaller, L. Schellenberg, E. B. Shera, and C. W. 
De Jager, Atom. Data Nucl. Data Tabl. 60, 177 (1995).

[9] J. Heisenberg and H. P. Blok, Ann. Rev. Nucl. Part. Sci. 33, 569 (1983).

[10] E. Moya de Guerra, Phys. Rept. 138, 293 (1986).

[11] M. N. Harakeh and A. van der Woude, Giant Resonances, Oxford Studies in Nuclear Physics, Vol. 24 (Clarendon Press, Oxford, 2001).

[12] S. Frullani and J. Mougey, Adv. Nucl. Phys. 14, 1 (1984).

[13] M. Bernheim, A. Bussiere, J. Mougey, D. Royer, S. Tarnowski, D. Turck-Chièze, S. Frullani, S. Boffi, C. Giusti, and F. D. Pacati, Nucl. Phys. A375, 381 (1982).

[14] L. Lapikás, Nucl. Phys. A553, 297 (1993).

[15] P. K. A. de Witt Huberts, J. Phys. G16, 507 (1990).

[16] J. M. Udías, P. Sarriguren, E. Moya de Guerra, E. Garrido, and J. A. Caballero, Phys. Rev. C 48, 2731 (1993).

[17] J. Gao et al. (The Jefferson Lab Hall A Collaboration), Phys. Rev. Lett. 84, 3265 (2000).

[18] http://faculty.virginia.edu/qes-archive/index.html.

[19] I. Tanhihata, Prog. Part. Nucl. Phys. 35, 505 (1995).

[20] H. Geissel, G. Müzenberg, and R. Riisager, Ann. Rev. Nucl. Part. Sci. 45, 163 (1995).

[21] A. Mueller, Prog. Part. Nucl. Phys. 46, 359 (2001).

[22] T. Suda, K. Maruyama, and I. Tanhihata, RIKEN Accel. Prog. Rep. 34, 49 (2001).

[23] T. Suda, M. Wakasugi, T. Emoto, K. Ishii, S. Ito, K. Kurita, A. Kuwajima, A. Noda, T. Shirai, T. Tamae, H. Tongu, S. Wang, and Y. Yano, Phys. Rev. Lett. 102, 102501 (2009).

[24] T. Katayama, T. Suda, and I. Tanhihata, Physica Scripta T104, 129 (2003).

[25] "An International Accelerator Facility for Beams of Ions and Antiprotons", GSI report 2006, http://www.gsi.de/GSI-Future/cdr/.

[26] http://www.gsi.de/forschung/fair_experiments/elise/index_e.html.

[27] H. Simon, Nucl. Phys. A787, 102 (2007).

[28] A. N. Antonov et al., Nucl. Instrum. Meth. A637, 60 (2011).

[29] T. Suda, "A construction proposal of an electron scattering facility for structure studies of short-lived nuclei", Proposal for Nuclear Physics Experiments at RIBF NP1006 - SCRIT01 (2010).

[30] T. Suda, T. Adachi, T. Amagai, A. Enokizono, M. Hara, T. Hori, S. Ichikawa, K. Kurita, 
T. Miyamoto, R. Ogawara, T. Ohnishi, Y. Shimakura, T. Tamae, M. Togasaki, M. Wakasugi, S. Wang, and K. Yanagi, Progress of Theoretical and Experimental Physics 2012, 03C008 (2012).

[31] E. Garrido and E. Moya de Guerra, Nucl. Phys. A650, 387 (1999).

[32] E. Garrido and E. Moya de Guerra, Phys. Lett. B488, 68 (2000).

[33] S. Ershov, B. Danilin, and J. Vaagen, Phys. Rev. C 72, 044606 (2005).

[34] Z. Wang and Z. Ren, Phys. Rev. C 70, 034303 (2004).

[35] A. N. Antonov, M. K. Gaidarov, D. N. Kadrev, P. E. Hodgson, and E. Moya de Guerra, Int. J. Mod. Phys. E 13, 759 (2004).

[36] A. N. Antonov, D. N. Kadrev, M. K. Gaidarov, E. Moya de Guerra, P. Sarriguren, J. M. Udías, V. K. Lukyanov, E. V. Zemlyanaya, and G. Z. Krumova, Phys. Rev. C 72, 044307 (2005).

[37] C. A. Bertulani, Phys. Rev. C 75, 024606 (2007).

[38] E. Khan, M. Grasso, J. Margueron, and N. Van Giai, Nucl. Phys. A800, 37 (2008).

[39] M. Grasso, L. Gaudefroy, E. Khan, T. Nikšić, J. Piekarewicz, O. Sorlin, N. Van Giai, and D. Vretenar, Phys. Rev. C 79, 034318 (2009).

[40] Y. Chu, Z. Ren, T. Dong, and Z. W. Wang, Phys. Rev. C 79, 044313 (2009).

[41] X. Roca-Maza, M. Centelles, F. Salvat, and X. Viñas, Phys. Rev. C 78, 044332 (2008), 0808.1252 .

[42] X. Roca-Maza, M. Centelles, F. Salvat, and X. Viñas, Phys. Rev. C 87, 014304 (2013).

[43] T. Dong, Y. Chu, Z. Ren, and Z. Wang, Phys. Rev. C 79, 014317 (2009).

[44] T. Dong, Z. Ren, and Z. Wang, Phys. Rev. C 77, 064302 (2008).

[45] J. Liu, Z. Ren, and T. Dong, Nucl. Phys. A888, 45 (2012).

[46] T. Dong, Y. Chu, and Z. Ren, J. Phys. Conf. Ser. 381, 012135 (2012).

[47] C. Giusti, A. Meucci, F. D. Pacati, G. Co', and V. De Donno, Phys. Rev. C 84, 024615 (2011).

[48] G. Co', V. De Donno, P. Finelli, M. Grasso, M. Anguiano, A. M. Lallena, C. Giusti, A. Meucci, and F. D. Pacati, Phys. Rev. C 85, 024322 (2012).

[49] T. W. Donnelly, J. Dubach, and I. Sick, Nuclear Physics A 503, 589 (1989).

[50] T. W. Donnelly and R. D. Peccei, Physics Reports 50, 1 (1979).

[51] C. J. Horowitz, Phys. Rev. C 57, 3430 (1998). 
[52] S. Abrahamyan et al. (PREX Collaboration), Phys. Rev. Lett. 108, 112502 (2012).

[53] C. J. Horowitz, Z. Ahmed, C. M. Jen, A. Rakhman, P. A. Souder, M. M. Dalton, N. Liyanage, K. D. Paschke, K. Saenboonruang, R. Silwal, G. B. Franklin, M. Friend, B. Quinn, K. S. Kumar, D. McNulty, L. Mercado, S. Riordan, J. Wexler, R. W. Michaels, and G. M. Urciuoli, Phys. Rev. C 85, 032501 (2012).

[54] PREX-II, Proposal to Jefferson Lab PAC 38, http://hallaweb.jlab.org/parity/prex/ prexII.pdf.

[55] "CREX: parity-violating measurements of the weak charge distribution of ${ }^{48} \mathrm{Ca}$ to $0.03 \mathrm{fm}$ accuracy", Proposal to Jefferson Lab PAC 39, http://hallaweb.jlab.org/parity/prex.

[56] O. Moreno, P. Sarriguren, E. Moya de Guerra, J. M. Udías, T. W. Donnelly, and I. Sick, Nuclear Physics A 828, 306 (2009).

[57] S. Ban, C. J. Horowitz, and R. Michaels, Journal of Physics G: Nuclear and Particle Physics 39, 015104 (2012).

[58] X. Roca-Maza, M. Centelles, X. Viñas, and M. Warda, Phys. Rev. Lett. 106, 252501 (2011).

[59] E. Moya de Guerra, O. Moreno, P. Sarriguren, and M. Ramon, Journal of Physics: Conference Series 366, 012011 (2012).

[60] D. Vretenar, P. Finelli, A. Ventura, G. A. Lalazissis, and P. Ring, Phys. Rev. C 61, 064307 (2000).

[61] C. J. Horowitz, S. J. Pollock, P. A. Souder, and R. Michaels, Phys. Rev. C 63, 025501 (2001).

[62] P. Finelli, N. Kaiser, D. Vretenar, and W. Weise, Nuclear Physics A 735, 449 (2004).

[63] P. Finelli, N. Kaiser, W. Weise, and D. Vretenar, "Nuclear density functional constrained by low-energy QCD," in Theoretical Nuclear Physics In Italy, edited by S. Boffi, A. Covello, M. Di Toro, A. Fabrocini, G. Pisent, and S. Rosati (World Scientific, Singapore, 2004) Chap. 5, pp. 45-52.

[64] P. Finelli, N. Kaiser, D. Vretenar, and W. Weise, Nucl. Phys. A770, 1 (2006).

[65] C. Fuchs, H. Lenske, and H. H. Wolter, Phys. Rev. C 52, 3043 (1995).

[66] S. Typel and H. H. Wolter, Nuclear Physics A 656, 331 (1999).

[67] T. Nikšić, D. Vretenar, P. Finelli, and P. Ring, Phys. Rev. C 66, 024306 (2002).

[68] G. A. Lalazissis, T. Nikšić, D. Vretenar, and P. Ring, Phys. Rev. C 71, 024312 (2005).

[69] D. Vretenar, A. V. Afanasjev, G. A. Lalazissis, and P. Ring, Physics Reports 409, 101 
$(2005)$

[70] A. Meucci, F. Capuzzi, C. Giusti, and F. D. Pacati, Phys. Rev. C 67, 054601 (2003).

[71] A. Meucci, C. Giusti, and F. D. Pacati, Nuclear Physics A 739, 277 (2004).

[72] A. Meucci, C. Giusti, and F. D. Pacati, Nuclear Physics A 756, 359 (2005).

[73] A. Meucci, J. A. Caballero, C. Giusti, F. D. Pacati, and J. M. Udías, Phys. Rev. C 80, 024605 (2009).

[74] A. Meucci, J. A. Caballero, C. Giusti, and J. M. Udías, Phys. Rev. C 83, 064614 (2011).

[75] A. Meucci and C. Giusti, Phys. Rev. D 85, 093002 (2012).

[76] A. Meucci, C. Giusti, and F. D. Pacati, Phys. Rev. D 84, 113003 (2011).

[77] A. Meucci, M. B. Barbaro, J. A. Caballero, C. Giusti, and J. M. Udías, Phys. Rev. Lett. 107, 172501 (2011).

[78] C. Giusti, A. Meucci, F. D. Pacati, G. Co', and V. De Donno, Journal of Physics: Conference Series 366, 012019 (2012).

[79] O. Moreno, P. Sarriguren, E. Moya de Guerra, J. M. Udías, T. W. Donnelly, and I. Sick, Journal of Physics: Conference Series 312, 092044 (2011).

[80] P. Moller and J. R. Nix, Nucl. Phys. A536, 20 (1992).

[81] A. Schiller, T. Baumann, J. Dietrich, S. Kaiser, W. Peters, and M. Thoennessen, Phys. Rev. C 72, 037601 (2005).

[82] A. Meucci, C. Giusti, and F. D. Pacati, Phys. Rev. C 64, 014604 (2001).

[83] A. Meucci, C. Giusti, and F. D. Pacati, Phys. Rev. C 64, 064615 (2001).

[84] A. Meucci, Phys. Rev. C 65, 044601 (2002).

[85] M. Radici, A. Meucci, and W. H. Dickhoff, Eur. Phys. J. A 17, 65 (2003).

[86] T. Tamae, Y. Sato, T. Yokokawa, Y. Asano, M. Kawabata, O. Konno, I. Nakagawa, I. Nishikawa, K. Hirota, H. Yamazaki, R. Kimura, H. Miyase, H. Tsubota, C. Giusti, and A. Meucci, Phys. Rev. C 80, 064601 (2009).

[87] C. Maieron, M. C. Martinez, J. A. Caballero, and J. M. Udías, Phys. Rev. C 68, 048501 (2003).

[88] A. Meucci, C. Giusti, and F. D. Pacati, Nuclear Physics A 744, 307 (2004).

[89] A. Meucci, C. Giusti, and F. D. Pacati, Acta Phys. Polon. B 37, 2279 (2006).

[90] A. Meucci, C. Giusti, and F. D. Pacati, Nuclear Physics A 773, 250 (2006).

[91] J. A. Caballero, Phys. Rev. C 74, 015502 (2006). 
[92] A. Meucci, C. Giusti, and F. D. Pacati, Phys. Rev. C 77, 034606 (2008).

[93] C. Giusti, A. Meucci, and F. D. Pacati, Acta Phys. Polon. B 40, 2579 (2009).

[94] J. A. Caballero, M. C. Martinez, J. L. Herraiz, and J. M. Udías, Phys. Lett. B 688, 250 $(2010)$.

[95] A. V. Butkevich, Phys. Rev. C 82, 055501 (2010).

[96] A. V. Butkevich, Phys. Rev. C 85, 065501 (2012).

[97] F. Capuzzi, C. Giusti, and F. D. Pacati, Nuclear Physics A 524, 681 (1991).

[98] F. Capuzzi, C. Giusti, F. D. Pacati, and D. N. Kadrev, Annals of Physics (N.Y.) 317, 492 (2005).

[99] Brookhaven National Laboratory - National Nuclear Data Center, http://www.nndc.bnl. gov/.

[100] P. Sarriguren, M. K. Gaidarov, E. Moya de Guerra, and A. N. Antonov, Phys. Rev. C 76, $044322(2007)$.

[101] Z. Wang and Z. Ren, Phys. Rev. C 71, 054323 (2005).

[102] E. D. Cooper, S. Hama, and B. C. Clark, Phys. Rev. C 80, 034605 (2009).

[103] T. de Forest Jr., Nuclear Physics A 392, 232 (1983).

[104] M. Anghinolfi, M. Battaglieri, N. Bianchi, R. Cenni, P. Corvisiero, A. Fantoni, P. Levi Sandri, A. Longhi, V. Lucherini, V. I. Mokeev, V. Muccifora, E. Polli, A. Reolon, G. Ricco, M. Ripani, P. Rossi, S. Simula, M. Taiuti, A. Teglia, and A. Zucchiatti, Nuclear Physics A 602, 405 (1996).

[105] C. F. Williamson, T. C. Yates, W. M. Schmitt, M. Osborn, M. Deady, P. D. Zimmerman, C. C. Blatchley, K. K. Seth, M. Sarmiento, B. Parker, Y. Jin, L. E. Wright, and D. S. Onley, Phys. Rev. C 56, 3152 (1997).

[106] M. B. Barbaro, J. A. Caballero, T. W. Donnelly, and C. Maieron, Phys. Rev. C 69, 035502 (2004).

[107] J. E. Amaro, M. B. Barbaro, J. A. Caballero, T. W. Donnelly, A. Molinari, and I. Sick, Phys. Rev. C 71, 015501 (2005).

[108] M. V. Ivanov, M. B. Barbaro, J. A. Caballero, A. N. Antonov, E. Moya de Guerra, and M. K. Gaidarov, Phys. Rev. C 77, 034612 (2008).

[109] G. Rufa, Nuclear Physics A 384, 273 (1982).

[110] see supplemental material at http://link.aps.org/supplemental/10.1103/ 
PhysRevLett.108.112502.

[111] B. B. P. Sinha, G. A. Peterson, R. R. Whitney, I. Sick, and J. S. McCarthy, Phys. Rev. C 7, 1930 (1973).

[112] I. Sick and J. S. McCarthy, Nucl. Phys. A150, 631 (1970). 

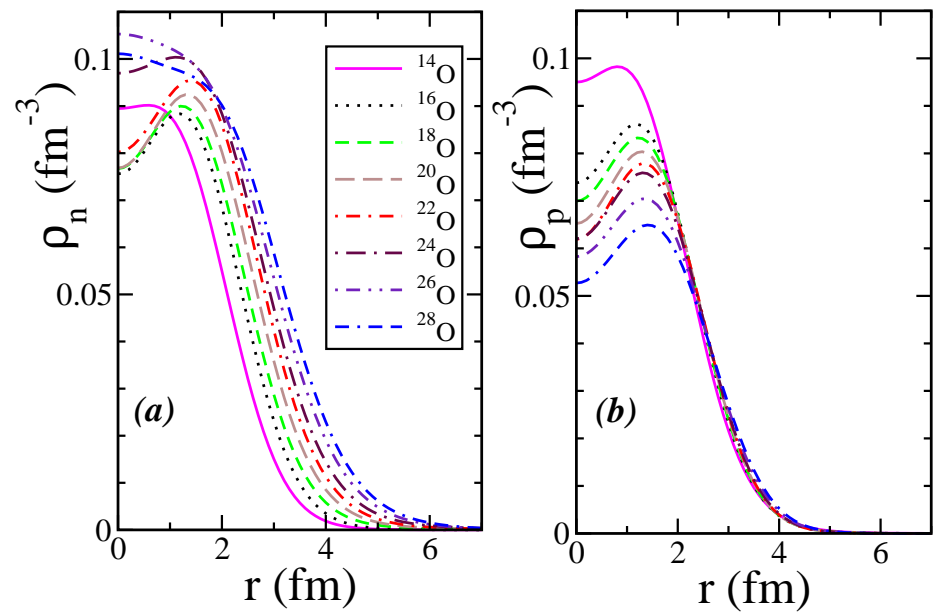

Figure 1. (Color online) Neutron, panel (a), and proton, panel (b), distributions for the various oxygen isotopes we have considered.
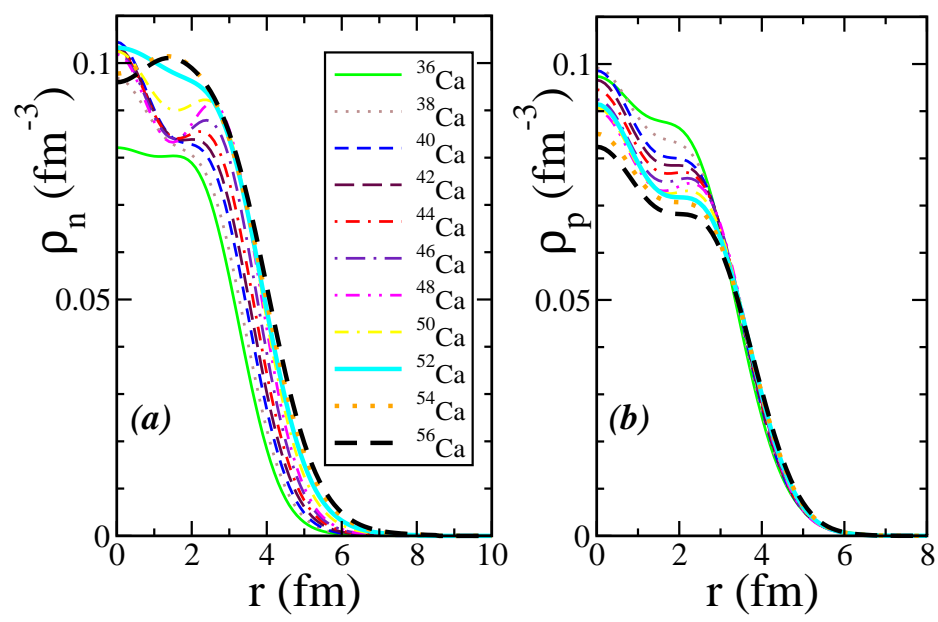

Figure 2. (Color online) The same as in Fig. 1, but for calcium isotopes. 


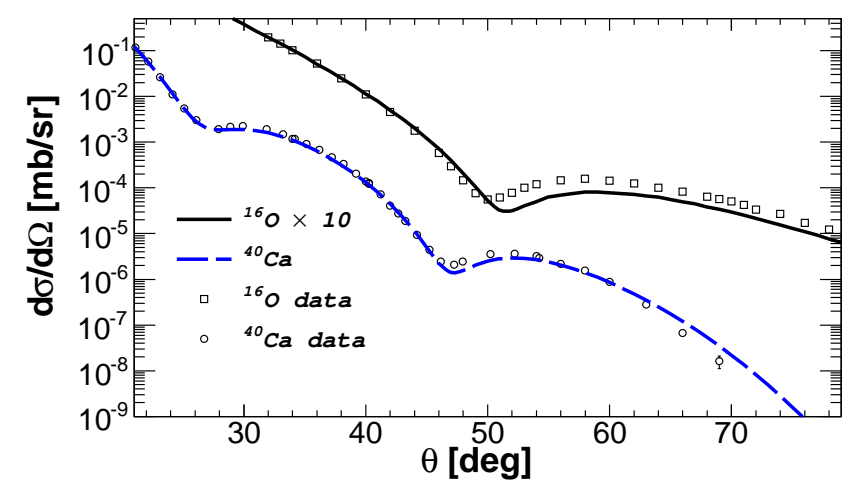

Figure 3. (Color online) Differential cross section for elastic electron scattering on ${ }^{16} \mathrm{O}$ at an electron energy $\varepsilon=374.5 \mathrm{MeV}$ and ${ }^{40} \mathrm{Ca}$ at $\varepsilon=496.8 \mathrm{MeV}$ as a function of the scattering angle $\theta$. Experimental data from $[111]\left({ }^{16} \mathrm{O}\right)$ and $[112]\left({ }^{40} \mathrm{Ca}\right)$.

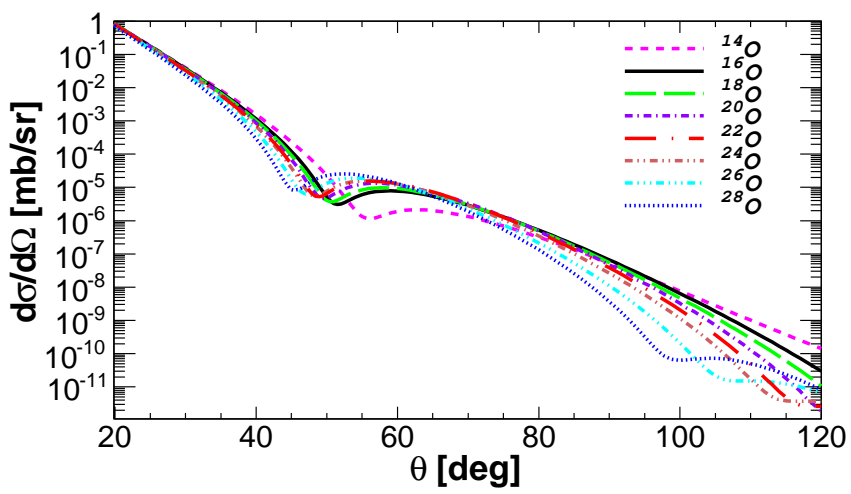

Figure 4. (Color online) Differential cross section for elastic electron scattering on ${ }^{14-28} \mathrm{O}$ at $\varepsilon=374.5 \mathrm{MeV}$ as a function of $\theta$. 


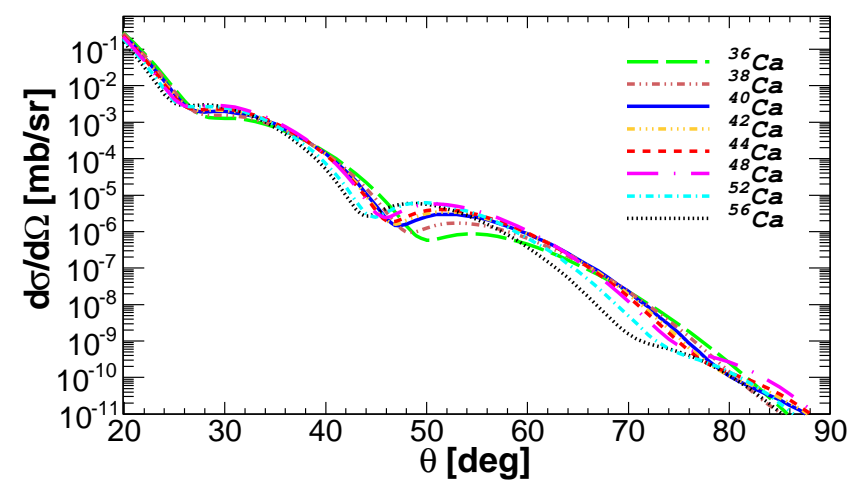

Figure 5. (Color online) Differential cross section for elastic electron scattering on ${ }^{36-56} \mathrm{Ca}$ at $\varepsilon=496.8 \mathrm{MeV}$ as a function of $\theta$.
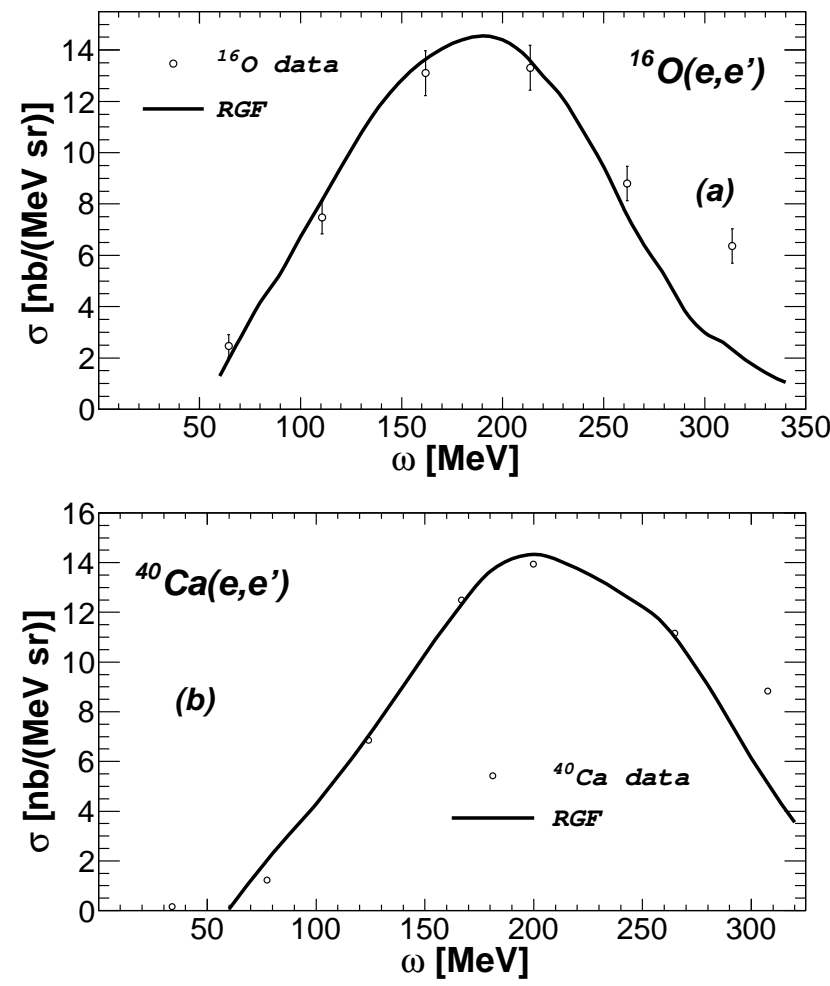

Figure 6. Differential cross section of the reactions ${ }^{16} \mathrm{O}\left(e, e^{\prime}\right)$, panel (a), and ${ }^{40} \mathrm{Ca}\left(e, e^{\prime}\right)$, panel (b), for different beam energies and electron scattering angles, $\varepsilon=1080 \mathrm{MeV}$ and $\theta=32^{\circ}$ for ${ }^{16} \mathrm{O}\left(e, e^{\prime}\right)$ and $\varepsilon=841 \mathrm{MeV}$ and $\theta=45.5^{\circ}$ for ${ }^{40} \mathrm{Ca}\left(e, e^{\prime}\right)$, as a function of the energy transfer $\omega$. The RGF results are compared with the experimental data from $[104]\left({ }^{16} \mathrm{O}\left(e, e^{\prime}\right)\right)$ and $[105]\left({ }^{40} \mathrm{Ca}\left(e, e^{\prime}\right)\right)$. 

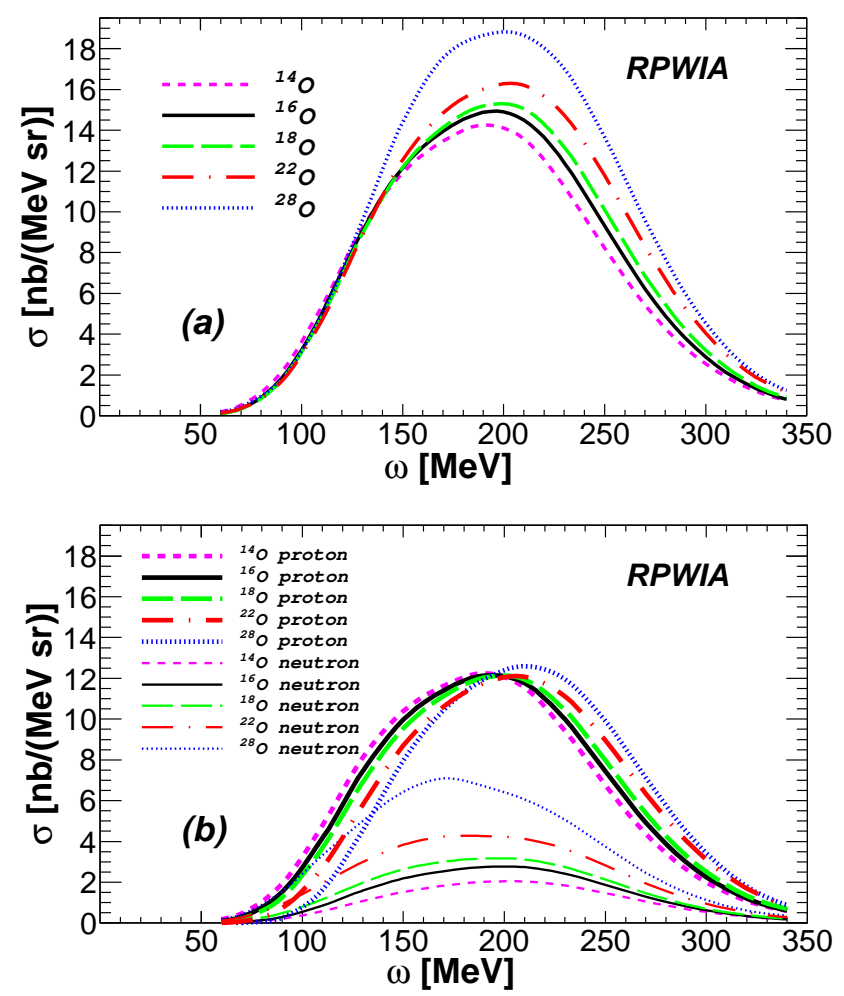

Figure 7. (Color online) In panel (a) the differential RPWIA cross section for the inclusive QE $\left(e, e^{\prime}\right)$ reaction on ${ }^{14-28} \mathrm{O}$ at $\varepsilon=1080 \mathrm{MeV}$ and $\theta=32^{\circ}$ is shown as a function of $\omega$. In panel (b) the separate contributions of protons (thick lines) and neutrons (thin lines) are displayed.

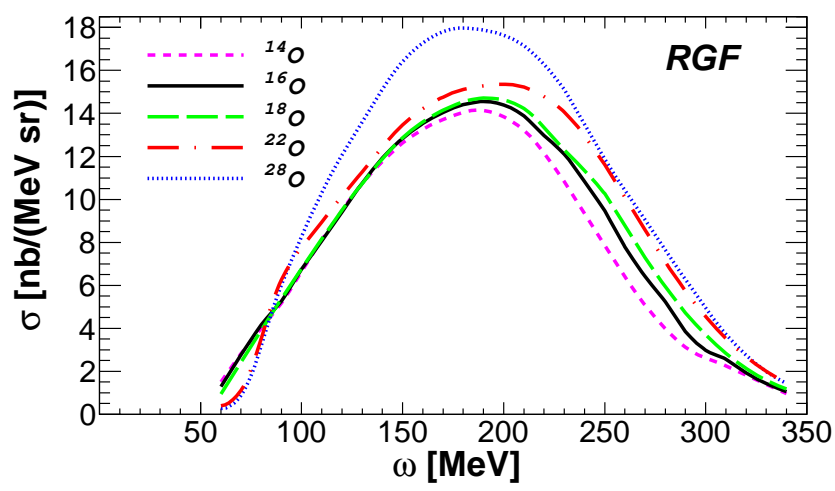

Figure 8. (Color online) Differential RGF cross section for the inclusive QE $\left(e, e^{\prime}\right)$ reaction on ${ }^{14-28} \mathrm{O}$ in the same kinematics as in Fig. 7. 


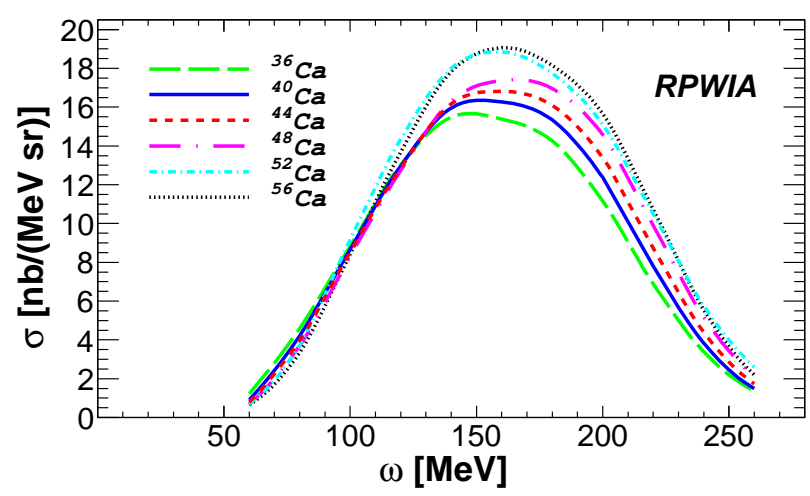

Figure 9. (Color online) Differential RPWIA cross section for the inclusive QE $\left(e, e^{\prime}\right)$ reaction on ${ }^{36-56} \mathrm{Ca}$ at $\varepsilon=560 \mathrm{MeV}$ and $\theta=60^{\circ}$ as a function of $\omega$.

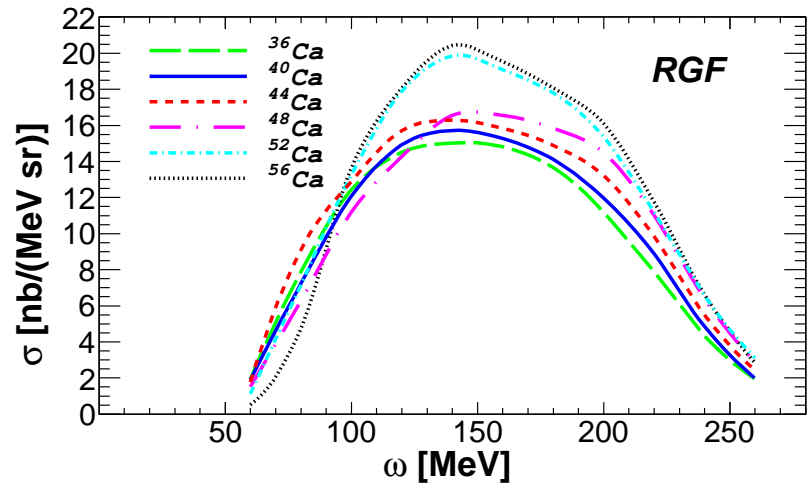

Figure 10. (Color online) The same as in Fig. 9, but in the RGF model. 


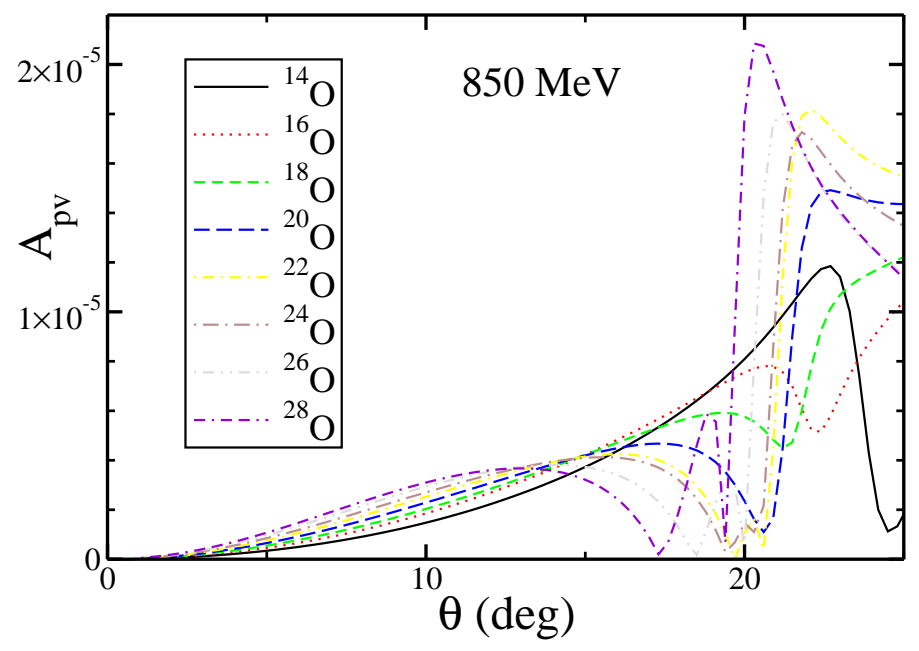

Figure 11. (Color online) Parity violating asymmetry parameter $A_{p v}$ for elastic electron scattering at $\varepsilon=850 \mathrm{MeV}$ as function of the scattering angle $\theta$ on ${ }^{14-28} \mathrm{O}$.

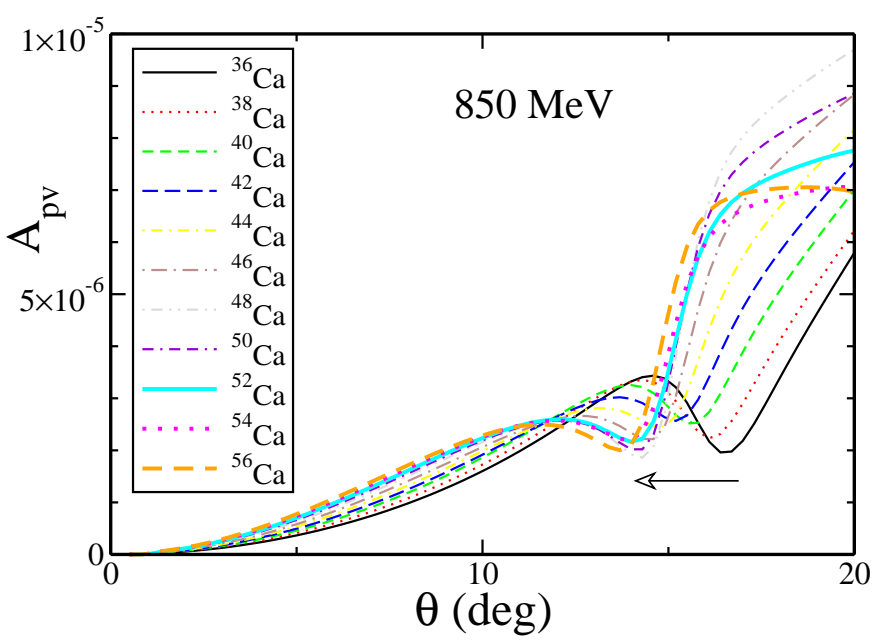

Figure 12. (Color online) The same as in Fig. 11, but for ${ }^{36-56}$ Ca. The black arrow emphasizes the evolution of $A_{p v}$ as a function of the neutron number $N$. 


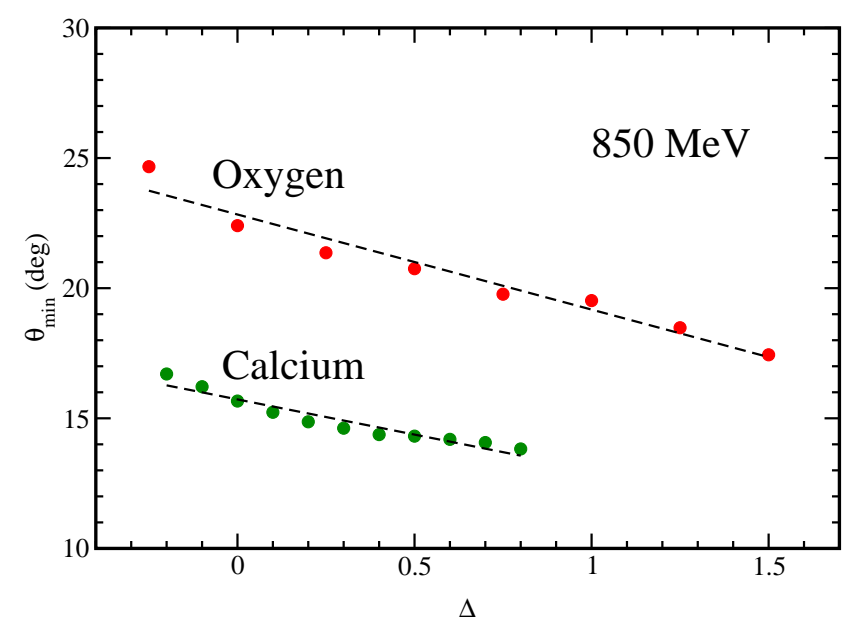

Figure 13. (Color online) First minimum positions of the asymmetry parameter $A_{p v}$ as functions of $\Delta=(N-Z) / Z$ for ${ }^{14-28} \mathrm{O}$ and ${ }^{36-56} \mathrm{Ca}$. The black dashed lines represent the best linear fit.
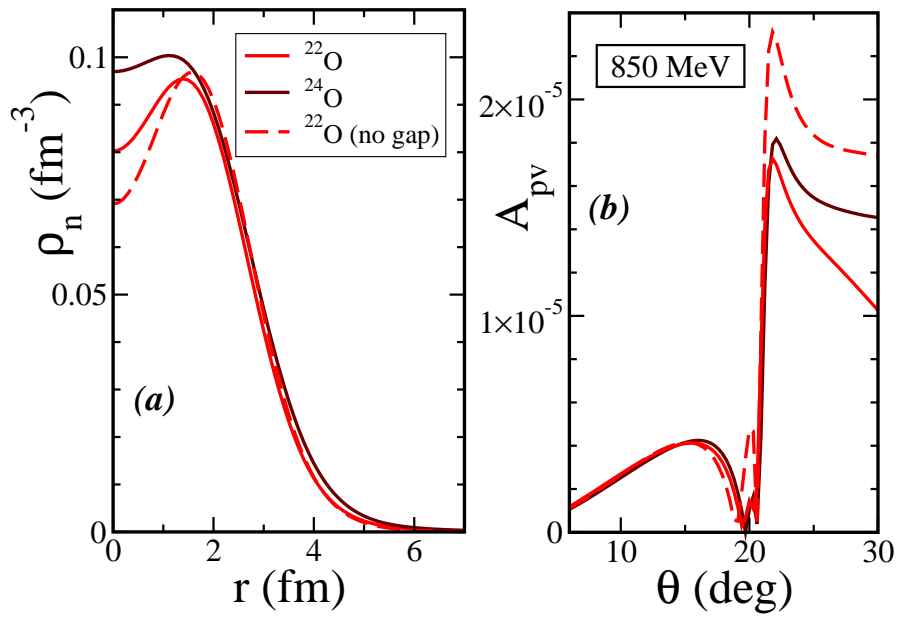

Figure 14. (Color online) Panel (a): neutron density distributions for some selected nuclei $\left({ }^{22} \mathrm{O}\right.$ and ${ }^{24} \mathrm{O}$ ) that could be interpreted as candidates for "bubble" structure [39]. Panel (b): parity violating asymmetry parameter $A_{p v}$ for elastic electron scattering at $\varepsilon=850 \mathrm{MeV}$ as a function of the scattering angle $\theta$. 

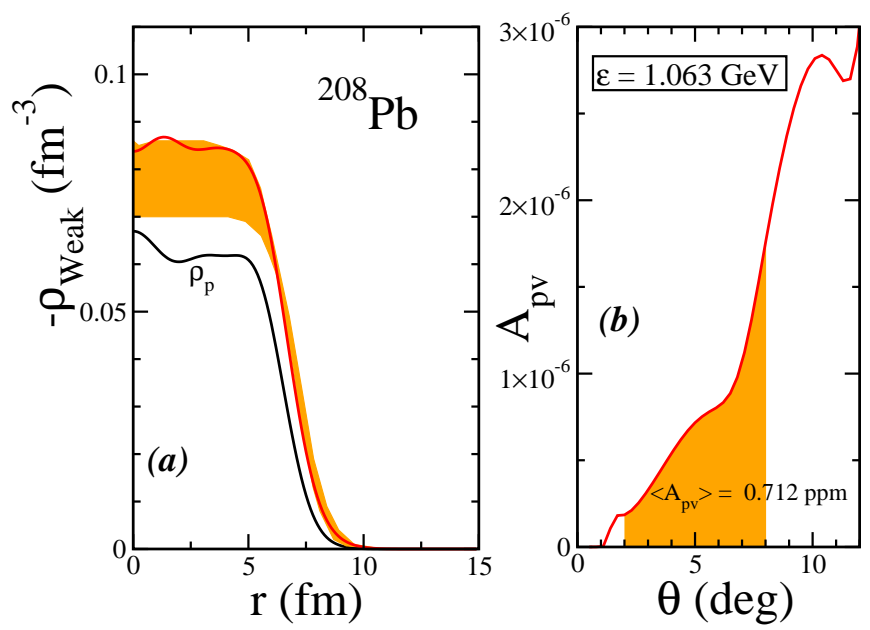

Figure 15. (Color online) Panel (a): Theoretical weak charge density (red line) in comparison with the experimental error band as determined in Ref. [53] for ${ }^{208} \mathrm{~Pb}$ with the kinematics of the PREX experiment. The corresponding proton density profile is plotted with a black line. Panel (b): Asymmetry parameter for ${ }^{208} \mathrm{~Pb}$ as a function of the angle $\theta$ (red line). The shaded area represents the interval covered by the acceptance function $\epsilon(\theta)$ (see Ref. [52] for more details). The asymmetry parameter averaged over the acceptance $\left\langle A_{p v}\right\rangle$ is in quite good agreement with the empirical value $0.656 \pm 0.060$ (stat) \pm 0.014 (syst) $\mathrm{ppm}$.

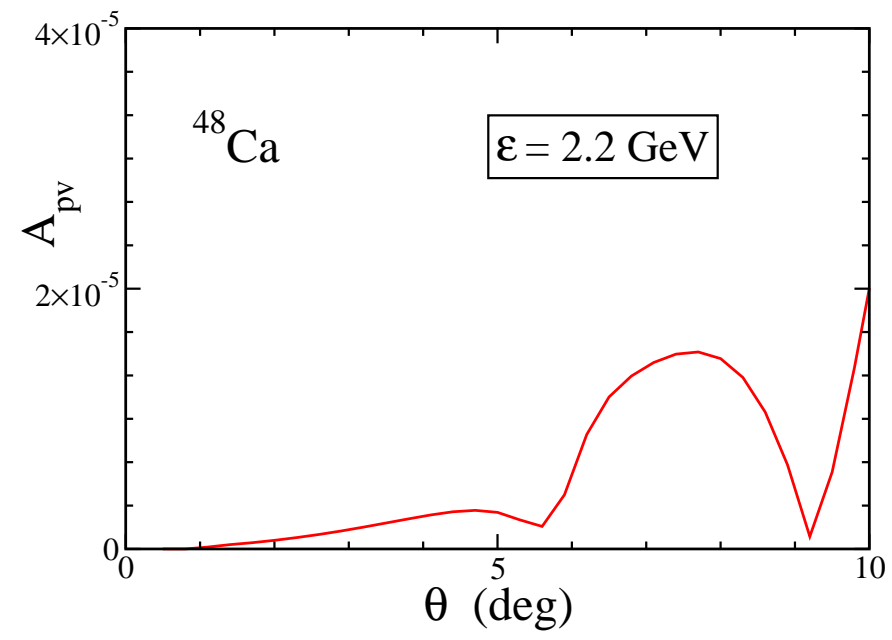

Figure 16. (Color online) Parity violating asymmetry parameters $A_{p v}$ for elastic electron scattering at $\varepsilon=2.2 \mathrm{GeV}$ as a function of the scattering angle $\theta$ for ${ }^{48} \mathrm{Ca}$, see Ref. [55]. 


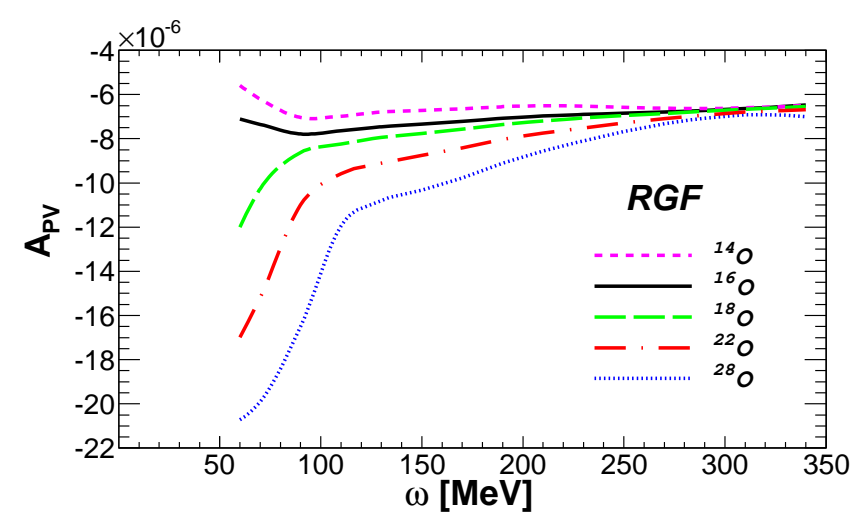

Figure 17. (Color online) Parity-violating asymmetry for the quasielastic $\left(e, e^{\prime}\right)$ reaction on $\mathrm{O}$ isotopes with the relativistic Green's function for $\varepsilon=1080 \mathrm{MeV}$ and $\theta=32^{\circ}$. 